\title{
The Human Preprotachykinin-A Gene Promoter Has Been Highly Conserved and Can Drive Human-like Marker Gene Expression in the Adult Mouse CNS
}

\author{
Alasdair MacKenzie, ${ }^{*}$ Catherine Payne,* Shelagh Boyle, ${ }^{+}$ \\ Alan R. Clarke, ${ }^{\ddagger}$ and J ohn P. Quinn*,1 \\ *Department of Veterinary Pathology, Royal (Dick) School of Veterinary Studies, \\ Summerhall Square, The University of Edinburgh, Edinburgh EH91QH, Scotland; ${ }^{+} M R C$ \\ Human Genetics Unit, Western General Hospital, Crewe Road, Edinburgh EH4 2XU, \\ Scotland; and ${ }^{\ddagger}$ Biomedical Sciences Building, Museum Avenue, The University of Cardiff, \\ Cardiff CF10 3US, Wales
}

\begin{abstract}
Toward an understanding of the mechanisms controlling Preprotachykinin-A (PPTA) transcription, we introduced a 380-kb human yeast artificial chromosome containing the PPTA gene tagged with the $\beta$-galactosidase gene into transgenic mice. This resulted in a pattern of Lac $Z$ expression in the central nervous system (CNS) remarkably similar to that reported for PPTA mRNA in the rat. However, the human gene drove expression in areas of the mouse CNS not associated with strong PPTA expression in rodents but which have been shown to express PPTA in the human. This study clearly demonstrates the high degree of conservation of the mechanisms involved in PPTA transcription that has occurred throughout 100 million of divergent human and rodent evolution. This study also defines the maximum linear extent of the human PPT-A promoter. We believe these findings constitute the removal of a significant obstacle in studying the transcriptional regulation of the human PPTA gene in vivo.
\end{abstract}

\section{INTRODUCTION}

Substance $\mathrm{P}(\mathrm{SP})$ is a biologically potent neuropeptide that has been shown to have an involvement in motor behavior, neuroendocrine function, and nociception (Maggio, 1988; Pernow, 1983). SP is produced from the preprotachykinin-A gene (PPTA), which also produces neurokinin-A (NKA), Neuropeptide-K, and neuropep-

\footnotetext{
${ }^{1}$ To whom correspondence and reprint requests should be addressed. E-mail: jquinn@vet.ed.ac.uk.
}

tide- $\gamma$ (Bannon et al., 1992; Krause et al., 1987). The removal of sequences encoding substance $\mathrm{P}$ (SP) and neurokinin-A (NKA) from the preprotachykinin-A (PPTA) gene in mice significantly reduces their ability to experience moderate to intense pain and neurogenic inflammation. The results of SP/NKA removal demonstrates the functional importance of PPTA expression in the dorsal root ganglia and dorsal horns of the peripheral nervous system (PNS; Cao et al., 1998; Zimmer et al., 1998).

In addition to the PNS, PPTA mRNA has been localized to a diverse range of central nervous system (CNS) structures in the rat. These areas include the striatum, olfactory tubercle (particularly the Islands of Calleja), cerebral cortex (particularly the cingulate cortex), superior colliculus, amygdala, medial haberacular nucleus, substantia nigra, hypothalamus, periaqueductal grey, and the zona incerta (Brene et al., 1990; Harlan et al., 1989; Warden and Young, 1988). In humans PPTA mRNA has also been found to be strongly expressed in areas such as the mammillary bodies of the hypothalamus and the dentate gyrus (Hurd et al., 1999). Several studies suggests a neuroprotective role for PPTA in the CNS in that SP can enhance neural growth in vitro (Iwasaki et al., 1989), can counteract the effects of neurotoxins administered to animals (Krasnova, 2000) and has mnemogenic and anxiolytic properties in vivo (Hasenohrl et al., 1998). Furthermore, SP/PPTA expression has also been studied in response to several neurodegenerative disorders. For example, Parkinsons dis- 
ease (Gresch and Walker, 1999) Alzheimers disease (Bouras et al., 1990) and Huntington disease (Richfield et al., 1995) are all associated with a progressive loss of PPTA and SP expression within the brain. This gene, therefore, exhibits both tissue specific and inducible expression, in the latter such regulated expression might be mechanistically correlated with progression of disease.

Many in vitro studies have been successful in demonstrating transcriptional activity around the proximal promoter (Fiskerstrand and Quinn, 1996; Fiskerstrand et al., 1999a,1999b; Mendelson et al., 1998; Morrison et al., 1994a,1994b; Quinn et al., 1995a,1995b). However, in vivo studies with as much as $10 \mathrm{~kb}$ of the rat PPTA proximal promoter have proven less successful (Harmar et al., 1993); Allan Basbaum, personal communication; Tony Harmar, personal communication). This indicates that elements influencing the expression of the PPTA gene may lie at some distance from the major start site of transcription. This is consistent with the promoters of several other genes. For example, elements regulating the expression of the tyrosine kinase gene were found to lie at $-12 \mathrm{~kb}$ (Montoliu et al., 1996), in the case of the Myf gene elements were found to lie between 50 and $95 \mathrm{~kb}$ of the gene start site (Zweigerdt et al., 1997). Furthermore, elements controlling the expression of the human $\beta$-globin gene were found at -60 $\mathrm{kb}$ (Porcu et al., 1997). With this in mind we set about introducing a human yeast artificial chromosome (YAC) into mice to produce an in vivo model of human PPTA expression (Lamb and Gearhart, 1995).

We have succeeded in generating mouse transgenic lines, which strongly and specifically expresses the bacterial $\beta$-galactosidase ( $\mathrm{LaCZ}$ ) gene in most if not all of the regions of the CNS that have been shown to express the PPTA gene by in situ hybridization in the adult rat (Brene et al., 1990; Harlan et al., 1989; Warden and Young, 1988). This study demonstrates that the molecular mechanisms involved in PPTA expression pattern generation have been strongly conserved throughout evolution between human, rat and mouse. Significantly, human specific expression patterns in the mammillary body, the dentate gyrus and the cerebellum are produced in areas of the transgenic CNS not normally associated with PPTA gene expression in rodents but which are associated with PPTA expression in humans.

\section{RESULTS}

The GenBank database (NIH) shows that, with the exception of the PPTA gene and the Asparagine syn- thase gene at the $3^{\prime}$ end of PAC clone AC005327, an area of sequence not included in the YAC (Fig. 1A), none of the PAC and BAC clones described in Fig. 1A contain any significant open reading frames or EST marker sites. We concluded from this that the chances of other active genes remaining undetected within the sequence of YAChPPTLacZ were remote. Flourescent in situ hybridization (FISH) analysis of human metaphase chromosome spreads using YAChPPTLacZ as probe demonstrated that YAChPPTLacZ was nonchimeric as only one signal was obtained within the nucleus at the correct locus (7q21.1; Figs. 2A and 2B). Two transgenic mouse lines were generated by oocyte microinjection (117 and 143) using YAChPPTLacZ DNA.

All the PCR primer pairs used for the analysis (Fig. 1A) gave positive signals for both lines through three generations of breeding the transgenic lines onto an MF1 mouse background (data not shown). Furthermore FISH analysis on several interphase spreads of transgenic mouse spleen cells showed only one integration site for the YAC in each transgenic line (Figs. 2C-2F). Although we accept that this data does not prove conclusively that shearing has not occurred during injection of the YAC it is consistent with the hypothesis that the injected YAC has remained intact.

Expression of the transgene was compared to rat PPTA mRNA expression, as opposed to immunohistochemical data, as peptides produced from the PPTA gene may be transported to areas of the brain some distance from their site of production. We have included adaptations of rat PPTA expression data for comparison because the rat is the most completely characterised model using mRNA in situ hybridization (Brene et al., 1990; Harlan et al., 1989; Warden and Young, 1988). In addition, all the literature describing PPTA mRNA or substance P expression in the mouse brain strongly suggests that little divergence of PPTA expression has occurred between mouse and rat brain in any of the areas described here (Airaksinen et al., 1992; Chesselet et al., 1987). Except where specifically mentioned the following description of the LacZ expression patterns obtained refer to both lines.

Forebrain. Expression of LacZ in both the anterior olfactory nucleus (Harlan et al., 1989) and the islands of Calleja of the olfactory tubercle (Fig. 3A) are entirely consistent with the published data in the rat (Fig. 3B; Brene et al., 1990; Harlan et al., 1989; Warden and Young, 1988). In addition, strong and consistent LacZ staining was observed in the caudate putamen (Fig. 3C), which bore a striking similarity to expression patterns previously reported (Fig. 3D; Brene et al., 1990; Harlan et al., 1989; Warden and Young, 1988). 


\section{A. YAChPPT6 and overlapping PAC/BAC Clones}

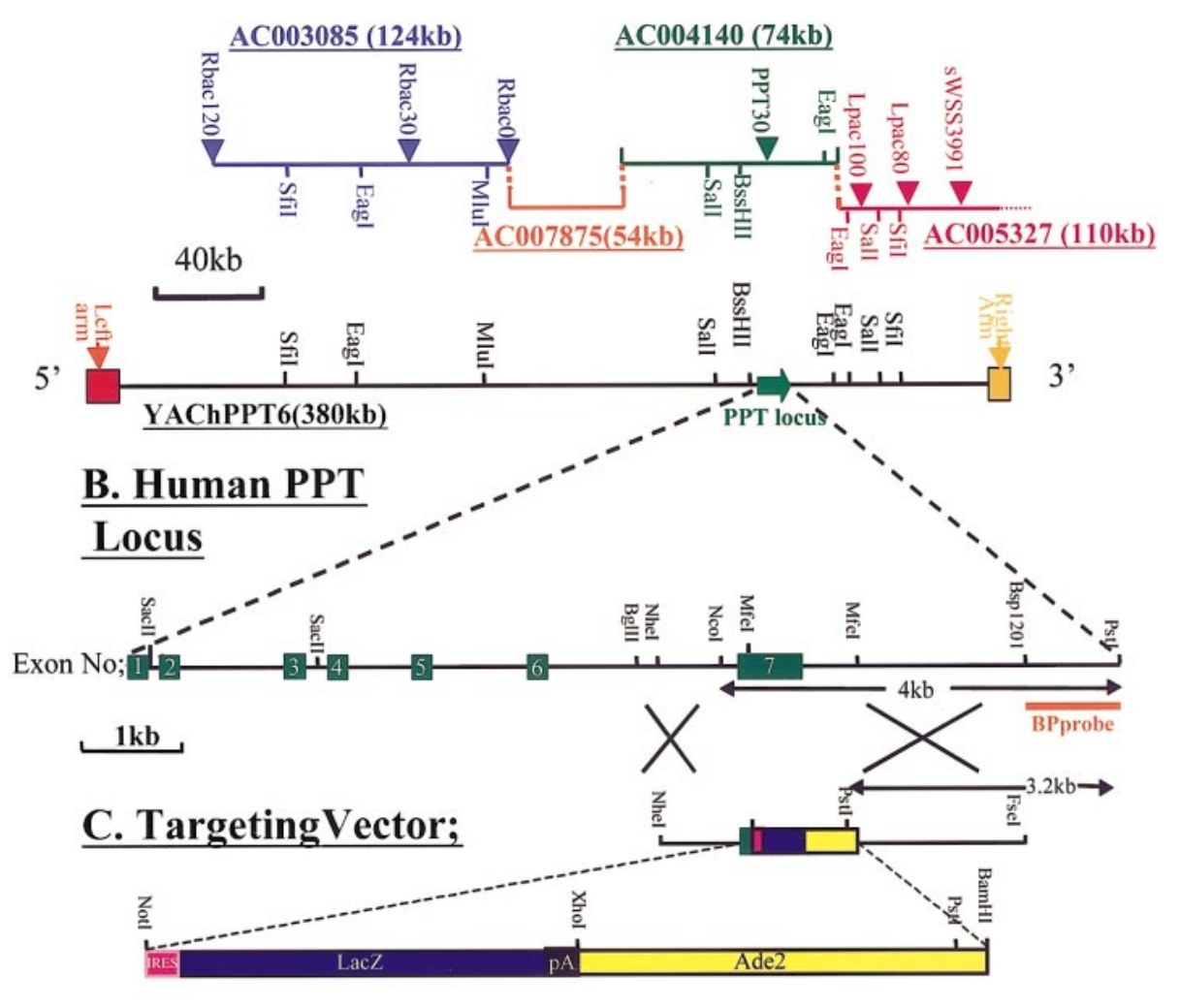

\section{Southern blot of yeast DNA digested with NcoI/PstI and probed with BPprobe}

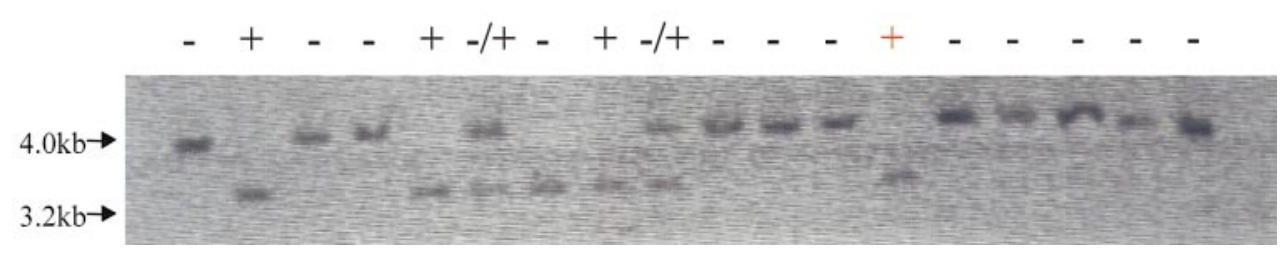

FIG. 1. (A) A representation of the linear relationships of the YAChPPT6 clone to sequenced human PAC and BAC clones showing several rare cutter restriction sites. PCR marker sites are shown as inverted solid triangles. (B) A representation of the expanded PPTA locus showing the location of each of the exons and their relationship to the restriction sites used for the manufacture of the targeting vector. The red line indicates the position of the BP probe used in D. (C) A line diagram representing the targeting vector used to introduce the LacZ gene into the YAChPPT6 clone. IRES, internal ribosome entry site; LacZ, $\beta$-galactosidase gene; pA, SV40 polyadenylation signal; Ade2, Yeast adenine marker gene. (D) An autoradiogram of a southern blot of NcoI- and PstI-digested yeast DNA hybridized to the BP probe (red line in B). Upper bands represent nonhomologous recombinants and lower bands represent homologous recombinant clones. The clone represented with a red cross was grown for YAC isolation for transgenic production.

The observed LacZ staining in the cortex and particularly the cingulate cortex (Figs. $3 \mathrm{C}$ and $3 \mathrm{E}$ ) are also consistent with rat PPTA mRNA expression previously described where PPTA transcripts were reported in the cerebral cortex of the rat (Figs. 3D and 3F; Warden and
Young, 1988) with distinctly higher transcript levels observed in the cingulate cortex (Brene et al., 1990).

Midbrain. LacZ staining was observed in the ventromedial and dorsomedial hypothalamus and the interpeduncular fossa where PPT mRNA has been previ- 

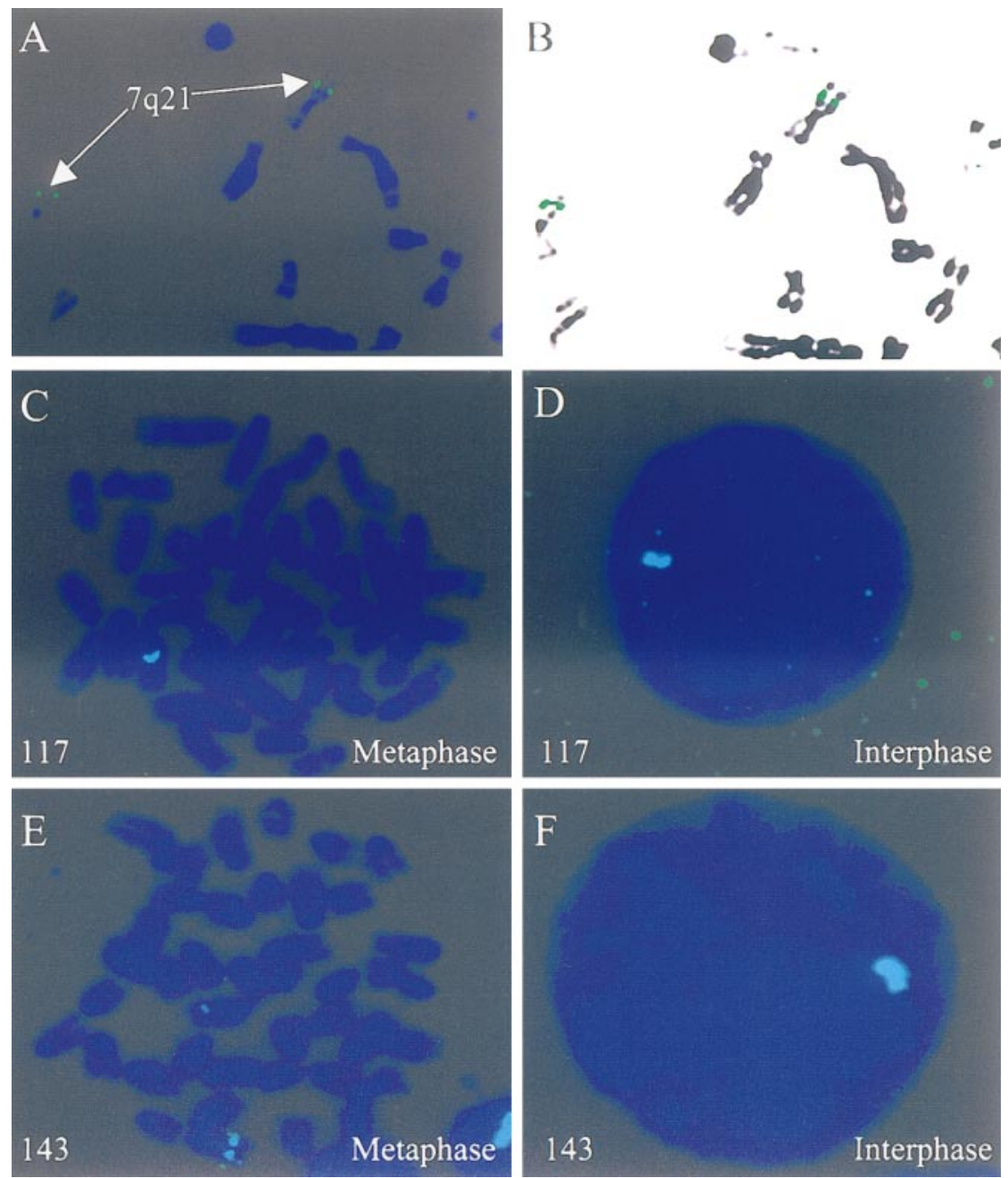

FIG. 2. (A and B) Dark field (A) and light field (B) photomicrographs of human metaphase chromosome preparations after FISH analysis with YAC hPPTLacZ DNA as probe showing a signal at position 7q21. (C-F) Dark field photomicrographs of mouse spleen cell chromosomes from transgenic lines 117 (C and D) and 143 (E and F) probed with YAChPPTLacZ showing signals obtained at metaphase (C and E) and interphase ( $\mathrm{E}$ and $\mathrm{F}$ ) to demonstrate the signal obtained in both condensed and uncondensed chromatin.

ously found in the rat (Figs. 3E and 3G and 4C; Warden and Young, 1988). However, expression in the rat mammillary body (Figs. 3G and 4C) was not mentioned in the rat literature.

LacZ staining in the superior colliculus (Figs. 3G and $3 \mathrm{H})$, the peri aqueductal grey, the medial habernacular nucleus (Figs. 3E and 3F) and the amygdaloid nucleii (Figs. 3E and $3 \mathrm{~F}$ ) occured in a similar pattern to that previously described for PPTA mRNA in the rat (Figs. 3G and 3H; Warden and Young, 1988). Substantia nigra was seen to stain strongly with LacZ in an area corresponding to the reticular substantia nigra, (Fig. 4A). This expression pattern was not reported by Warden and Young (1988) who make specific reference to a lack of expression in the rat substantia nigra. However, expression of PPTA transcripts in the rat substantia nigra is refered to by Brene et al. (1990).

LacZ was expressed in the pyramidal cell layer of the hippocampus of our human YAC transgenic model (Figs. 3E, 3F, and 4B), which is an area where PPTA transcripts are known to localise in the rat (Fig. 3F). However, our human YAC transgenic model also expresses LacZ within cells of the dentate gyrus (Fig. 4B) and the thalamus (Figs. 3E and 3G), areas of the physiologically normal rodent brain considered specifically bereft of transcripts by Warden and Young (1988).

Hindbrain. Expression of PPT was reported by both Warden and Young (1988) and Harlan et al. (1989) in the 
A

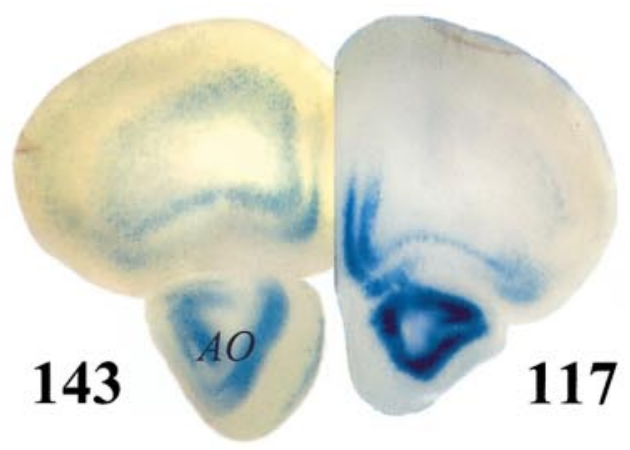

C

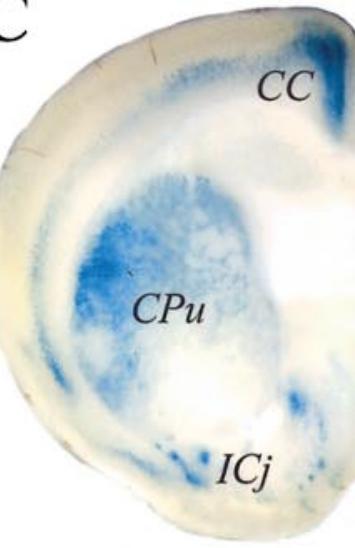

E
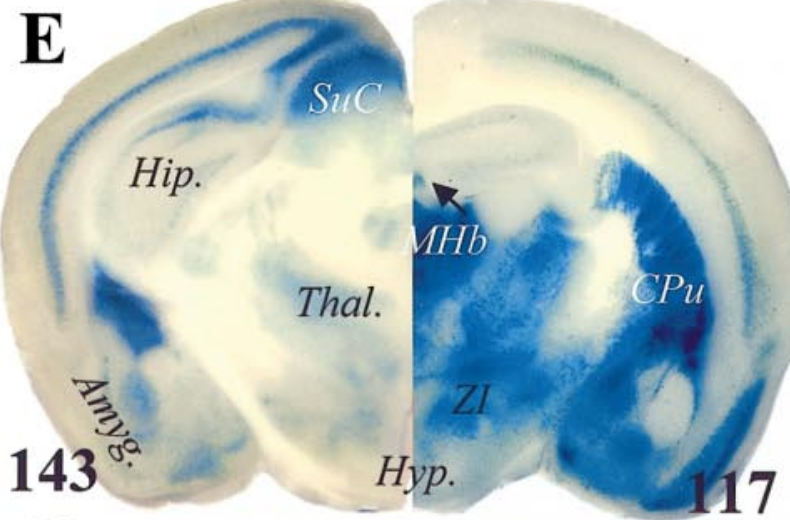

G

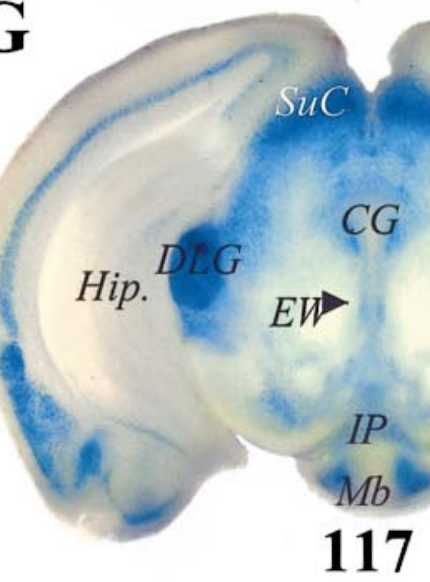

B

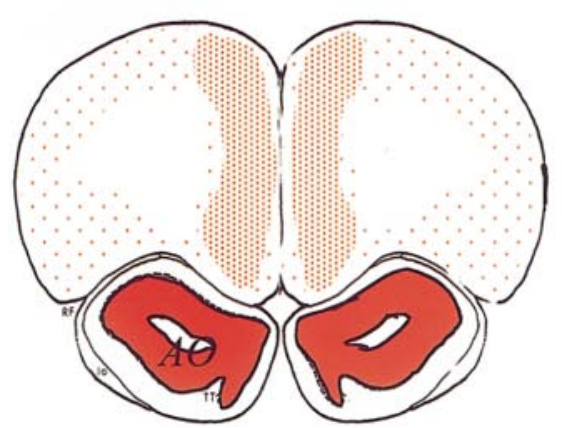

D

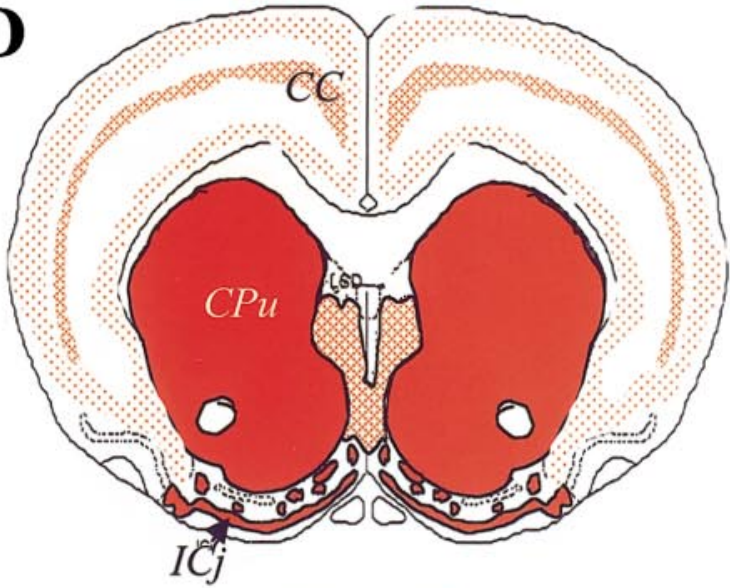

F

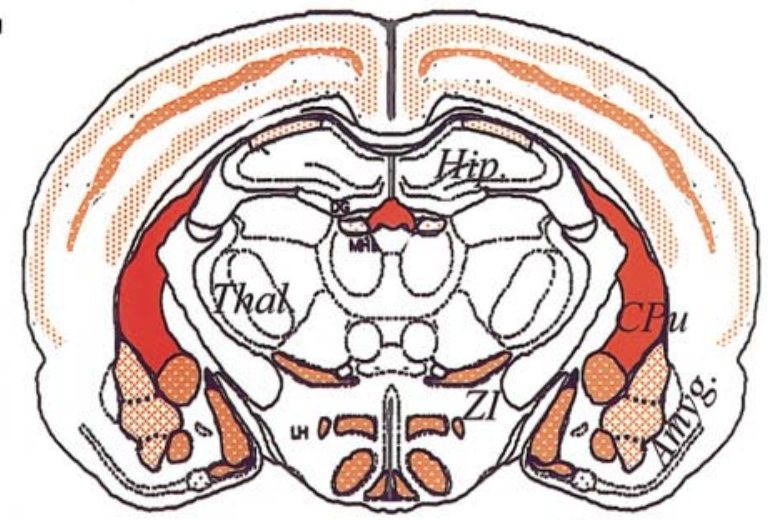

H

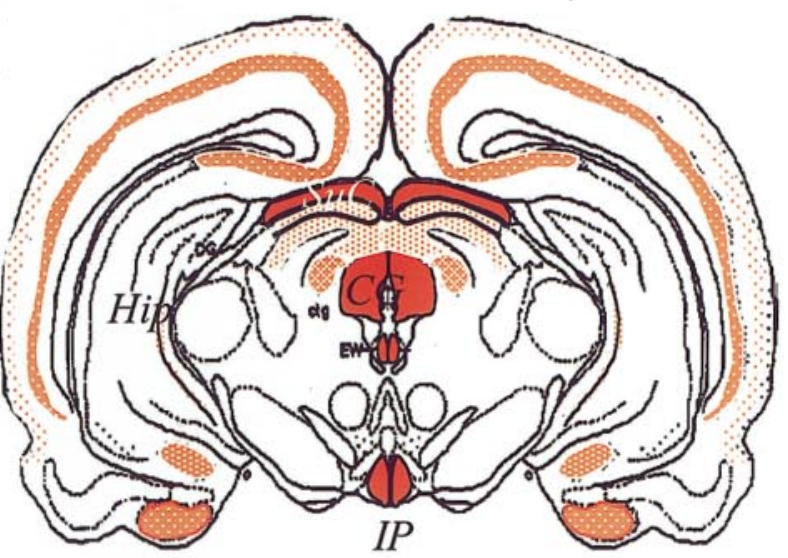


nucleus of the solitary tract in a pattern very similar to LacZ expression described here (Figs. 4D-4F). However, Strong LacZ staining was also observed in the cerebellum of both lines (Fig. 4E). This pattern occurred most strongly in line 143 but also occurred to a lesser degree in line 117 (Fig. 4E). None of the literature on expression of rat PPTA mRNA described high levels of expression in the rodent cerebellum (Brene et al., 1990; Harlan et al., 1989; Warden and Young, 1988).

Spinal cord and DRG. LacZ Staining was observed to occur in a subpopulation of cells within the soma of the DRG (Fig. 4G), which is consistent with published data (Noguchi et al., 1988).

Staining in the whole mount spinal cord occurred in two dorsal stripes that converged caudally throughout its length (Fig. 4H). When sectioned, LacZ staining in the spinal cord was observed to occur in lamina 1, 2, and 3 of the dorsal horn (Fig. 4I). This expression profile corresponds very well to the expression pattern of SP / PPTA mRNA within the spinal cord described in the literature (Fig. 4J; Harlan et al., 1989; Warden and Young, 1988).

\section{DISCUSSION}

\section{The Transcriptional Mechanisms Governing the Tissue-Specific Expression of the PPTA Gene in the Adult CNS Have Been Remarkably Conserved between Human, Mouse, and Rat}

There are a growing number of examples in the literature that contradicts the supposition that the promoters governing the expression of highly conserved genes have also been strongly conserved. For example several nonmouse gene promoters that have been introduced into transgenic mice which have not been able to direct appropriate expression of marker genes to the areas expected. These promoter elements include those of the human glucagon gene (Nian et al., 1999), the equine glycoprotein hormone $\alpha$-subunit gene (Farmerie et al., 1997), the human and chick type X-collagen gene (Beier et al., 1996), and a lamprey homeobox gene (Carr et al., 1998). We have used a 380-kb human YAC construct containing the PPT gene, tagged with the LacZ gene, to determine whether the promoter of the human PPTA gene could drive relevant expression of a marker gene within the mouse. Once transgenic mouse lines were constructed it became clear that the human gene could drive expression of the LacZ gene in the mouse in a pattern remarkably similar to that described for the rat (Brene et al., 1990; Harlan et al., 1989; Warden and Young, 1988).

The slight expression pattern differences shown between lines 117 and 143 may be a reflection of the random integration events of YAC DNA into separate foreign genomic sites. However, we have also observed that differences in expression patterns within each line are apparent between littermates (data not included). Therefore, differences between both lines may also represent the dynamic nature of human PPTA gene promoter activity. We have compared the expression of the transgene in two different lines to demonstrate that the patterns generated, while undoubtedly affected by their different sites of integration, remain highly representative of the known PPTA mRNA and SP expression patterns in the rat, human, and mouse as described above.

The clear conclusion of this study is that a high level of evolutionary conservation has occurred in the mechanisms governing the expression of the PPTA gene throughout the 100 million years of divergent evolution of mice, rats, and humans. We believe that this high degree of conservation, which was known to exist within the coding region of the PPTA gene and has now been demonstrated to exist within its promoter, underlines the importance of the products of the PPTA gene. Furthermore, we believe that the discovery that the human PPTA gene promoter can operate in the mouse is a major step forward in studying human PPTA transcriptional regulation in vivo. This trangenic model lays the foundation for definition of tissue specific and stimulus inducible expression in vivo by future deletion and mutation of the YAChPPTLacZ construct.

FIG. 3. (A, C, E, and G) Frontal vibratome sections of adult transgenic mouse brain demonstrating LacZ expression in various areas within the brains of mouse transgenic lines 143 and 117 (Fig. E143 has been cut at a slightly oblique angle compared to E117). (B, D, F and H) Line diagrams summarising the expression of PPTA mRNA (in red) in the rat brain (B; adapted from Harlan et al., 1989; D, F, and H; adapted from Warden and Young, 1988). AO, anterior olfactory nucleus; CC, cingulate cortex; Hip, hippocampus; CPu, caudate putamen; ICj, islands of Calleja; Thal, thalamus; Hyp, hypothalamus; SC, superior colliculus; Amgd, amygdala; ZI, zona incerta; MHb, medial habernicular nucleus; EW, EdingerWestphal nucleus; $I P$, interpeduncular nucleus; $C G$, central grey; DLG, dorsolateral genticulate nucleus. 

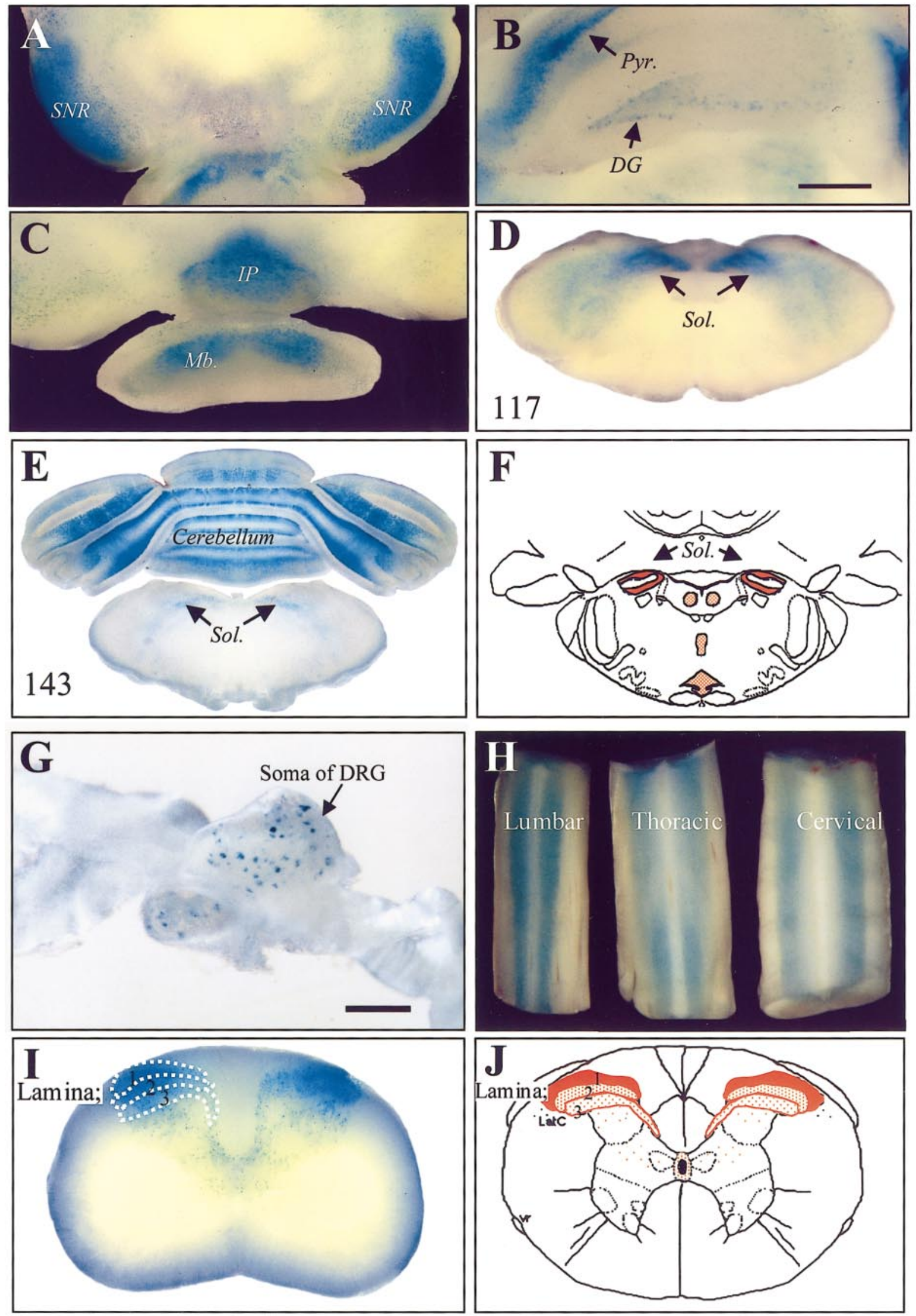


\section{The Human PPT Promoter Can Produce Ectopic "Human" PPT Expression Patterns in Areas of the Mouse CNS}

Although we have demonstrated that a high proportion of the expression patterns generated by the human YAC strongly resembles published rat PPTA mRNA expression certain LacZ patterns observed within the CNS do not resemble those of the rat. These include those found in the mammillary body, the cerebellum, and the dentate gyrus. Expression of LacZ in these areas may reflect the normal expression of PPTA in the mouse as distinct from rat. However, a recent study in the human brain specifically refers to a strong expression of PPTA in the human mammillary body and dentate gyrus (Hurd et al., 1999). We hypothesize that the expression of LacZ generated by the human PPTA containing YAC within the mammillary body and dentate gyrus of the mouse constitutes an ability of the human promoter to redirect expression of the marker gene to cells, within the mouse, that would not ordinarily express significant levels of PPTA. Moreover, studies have also noted that PPTA transcript levels in the cerebellum of Huntington disease victims are significantly elevated (Beal et al., 1988). The expression of LacZ in the cerebellum of our transgenic mice may reflect an aspect of human PPTA expression that may only occur in humans during pathogenesis. This strongly indicates that differences in the patterns of PPTA expression between species are a function of divergent evolution of promoter sequence in contrast to variations of transcription factors that would regulate promoter activity.

\section{The YAChPPTLacZ Construct Drives Expression in Areas Not Represented in the Published Data}

None of the rat studies mentioned above report of any PPT mRNA expression in the thalamus (Brene et al., 1990; Harlan et al., 1989; Warden and Young, 1988). In addition, only low levels of expression were reported in the human thalamus (Hurd et al., 1999). Without the supporting evidence demonstrating PPT expression in these areas it is difficult to reconcile these patterns with normal PPT promoter function. These patterns cannot represent integration effects as the same patterns were generated within both lines. They may, however, represent normal aspects of murine PPT expression. Another alternative, and more likely explanation, is that mRNA expression in these areas within the rat and human brain is normally so low as to be out with the detection limit of normal in situ hybridization.

\section{The Human YAC PPTA Transgenic System Can Be Used to Delineate Enhancer/Repressor Domains and the Roles of the PPTA Gene in the CNS during the Progression of Disease States}

We have defined the maximum linear extent of the human PPTA gene and its promoter responsible for controlling its expression within the CNS. It is highly likely that the minimal linear extent of the promoter will prove to be substantially smaller. We will systematically delete large sections of the YAC construct as described to delineate domains involved in the tissue specific and inducible expression of this gene as previously described (McKee-Johnson, 1996).

We have preliminary evidence suggesting that our lines contain up to four copies of the human PPTA gene. Furthermore, because the introduction of the $\beta$-galactosidase gene into the human PPTA only affected exon 7 , the integrity of much of the PPTA coding sequence, including those of exons 3 and 6, which encode for SP and NKA, respectively, remains. Current opinion within murine genetics now suggests that the over expression of genes may be very useful in determining gene function (Magdaleno and Curran, 1999). Therefore, we believe that these transgenic models will be invaluable in ascertaining the possible contributions of the PPTA gene in the production of several disease states. For example, recent studies have shown a strong correlation between the incidence of epilepsy, SP, and the integrity of the dentate gyrus in a rodent model (Liu et al., 1999; Liu, 2000). All these findings lead to the hypothesis that expression or addition of SP/PPTA in 
the rodent hippocampus and dentate gyrus, where it is not expressed under normal physiological conditions, results in seizure. How can this data be reconciled with the apparently contradictory observation that high levels of PPTA mRNA are expressed in the dentate gyrus of apparently healthy normal human subjects (Hurd et al., 1999)? We believe that the study of a mouse model of human PPTA expression may go some way in conciliating these bodies of data.

\section{EXPERIMENTAL METHODS}

Production of shuttle vector. A 3-kb SalI-PstI fragment containing exon 7 was isolated from a human EMBL3 SP6/T7 genomic $\lambda$-phage library and cloned into pGEM5z (Promega). A polylinker containing a NotI, XhoI, SacII, and BamHI sites (MWG Biotech) was cloned into the exon 7 sequence in place of a native 700-bp MfeI fragment (Fig. 1B). The yeast metabolic marker gene Ade2 was cloned into the SacII-BamHI sites of this polylinker (see Fig. 1C). The LacZ marker gene driven by a viral internal ribosome entry site (IRES) and terminating in a simian virus 40 (SV40) poly adenylation site was then cloned into the NotI-XhoI sites of the polylinker (see Fig. 1C). The shuttle vector was then released from its plasmid backbone by digestion with NheI and FseI.

Production of recombinant YAC (YAChPPTLacZ). A human ICI YAC library, obtained from the MRC HGMP resource center, was probed with human PPTA DNA. Three positive YAC clones containing the PPTA gene were detected and found to be nonchimeric by fluorescent in situ hybridization analysis on human chromosomes using the YAC clones as probes as described (Niederfuhr et al., 1998); Figs. 2A and 2B). Yeast clones containing the largest YAC were Lithium acetate transformed with the shuttle vector as described (McKee-Johnson, 1996) and clones which grew on SD Glucose medium lacking adenine were isolated (Markie, 1996). Homologous recombinant clones were determined by hybridisation of a radioactive probe to a nylon blot (Hybond, Amersham Pharmacia) endonuclease restricted Southern blotted total yeast DNA (see Fig. 1D).

Production of YAChPPTLacZ transgenic lines. YAChPPTLacZ DNA was isolated from yeast cultures as described (Schedl, 1996). Transgenic mice were generated as described (Hogan, 1995). YAChPPTLacZ DNA was microinjected at a concentration of $8 \mathrm{ng} / \mu \mathrm{l}$ into the pronucleii of one-cell embryos derived from superovulated CBAxC57BL/6 Fl females. Surviving in- jected eggs were transferred into pseudopregnant CD1 females.

PCR analysis of transgenic lines. Tail Tip biopsies were processed as described (MacKenzie and Quinn, 1999). Determination of YAC integrity was performed using a variety of PCR primer pairs on tail biopsy DNA samples. PCR detection of the YAC left and right arms (data not shown; Ragoussis, 1996) demonstrated integrity at the extreme limits of the YAC (Fig. 1A). In addition, several other primer pairs were used which confirmed the integrity of human sequences between the YAC arms. Internal human YAC sequence used to design these primers were derived from the known sequences of overlapping bacterial and P1 artificial chromosome clones (BAC and PAC; Fig. 1A).

The following primers were derived from these sequences using Vector NTI (f; forward primer, r; reverse primer; Fig. 1A): Left arm primer pair (Ragoussis, 1996): YACLR1, 5' GTG TGG TCG CCA TGA TCG CG 3'; YACLP, 5' ATG CGG TAG TTT ATC ACA GTT AA 3'; Primers derived from AC003085: Rbac120 f, 5'AGG AAA CCC AGG GCC TTT AAA A 3'; Rbac120 r, 5'GGG ACA AAA CAT CCA AAC CAG A 3'; Rbac30 f, 5'ATG GGC TTT CCT TTG AGG GTA A 3'; Rbac30 r, 5'GAA ATG AGC AAA GCC TCC AAG A 3'; Rbac0 f, 5'TTG AGG CTT GCA CCG TCT GA 3'; Rbac0 r, 5'TCC CCA GAG ATT TGC GGA A 3'; Primers derived from AC004140: PPT30 f, 5' CCA CCC CAC CAA TGC AGC T 3'; PPT30 r, 5' GGA GAG TGG GGT GAC CAG GTA AT 3'; Primers derived from AC005327: Lpac100 f, 5' ACA AGG AGA TGC TGT GGG GTG 3'; Lpac100 r, 5' TCC TCC AGG TGC ACA CTC TAC AC 3'; Lpac80 f, 5' TTA CCA GTC CAT GTC TGC ATT GTG 3'; Lpac80 r, 5' CCC AAA TCC GAA AGA CAG GAA A 3'; STS3991 f, 5' GAA ATA TCC TCT TGT TCA TC 3'; STS3991 r, 5' GAA TGT AAA GGT GTC TTA G 3'; Right arm primer pair (Ragoussis, 1996): YACRP, 5'GAT CAT CGT CGC GCT CCA AGC GAA AGC 3'; YACRR3,5' CTC GCC ACT TCG GGC TCA 3'.

LacZ analysis of transgenic lines. Mice of between 10 and 12 weeks of age were humanely sacrificed using $\mathrm{CO}_{2}$ and fixed by perfusion using $5 \%$ formaldehyde in phosphate-buffered saline (FPBS). Following dissection, whole brains, spinal cord, and DRG were fixed for $4 \mathrm{~h}$ in FPBS at $4^{\circ} \mathrm{C}$ until sufficiently firm for vibratome sectioning. Frontal sections $(200 \mu \mathrm{m})$ were LacZ stained as described (Hogan, 1995).

Flourescent in situ hybridization (FISH) analysis of mouse lines. Transgenic mice from both lines were humanely sacrificed and their spleens were placed in Dulbecco modified Eagles medium (DMEM) cell cul- 
ture medium. FISH analysis was carried out as described (Niederfuhr et al., 1998).

\section{ACKNOWLEDGMENTS}

The Medical Research Council funded this work. We thank San Bing Shen for technical advice and Gordon Arbuthnott for critically reading our manuscript. We also thank Christine Forrest, Billy Smith, and Brendan Doe for excellent technical support.

\section{REFERENCES}

Airaksinen, M. S., Alanen, S., Szabat, E., Visser, T. J., and Panula, P. (1992). Multiple neurotransmitters in the tuberomammillary nucleus: Comparison of rat, mouse, and guinea pig. J. Comp. Neurol. 323(1): 103-116.

Bannon, M. J., Poosch, M. S., Haverstick, D. M., Mandal, A., Xue, I. C., Shibata, K., and Dragovic, L. J. (1992). Preprotachykinin gene expression in the human basal ganglia: Characterization of mRNAs and pre-mRNAs produced by alternate RNA splicing. Brain Res. Mol. Brain Res. 12(1-3): 225-231.

Beal, M. F., Swartz, K. J., Finn, S. F., Bird, E. D., and Martin, J. B. (1988). Amino acid and neuropeptide neurotransmitters in Huntington's disease cerebellum. Brain Res. 454(1-2): 393-396.

Beier, F., Eerola, I., Vuorio, E., Luvalle, P., Reichenberger, E., Bertling, W., von der Mark, K., and Lammi, M. J. (1996). Variability in the upstream promoter and intron sequences of the human, mouse and chick type X collagen genes. Matrix Biol. 15(6): 415-422.

Bouras, C., Vallet, P. G., Hof, P. R., Charnay, Y., Golaz, J., and Constantinidis, J. (1990). Substance P immunoreactivity in Alzheimer disease: A study in cases presenting symmetric or asymmetric cortical atrophy. Alzheimer Dis. Assoc. Disord. 4(1): 24-34.

Brene, S., Lindefors, N., Friedman, W. J., and Persson, H. (1990). Preprotachykinin A mRNA expression in the rat brain during development. Brain Res. Dev. Brain Res. 57(2): 151-162.

Cao, Y. Q., Mantyh, P. W., Carlson, E. J., Gillespie, A. M., Epstein, C. J., and Basbaum, A. I. (1998). Primary afferent tachykinins are required to experience moderate to intense pain. Nature 392(6674): 390-394.

Carr, J. L., Shashikant, C. S., Bailey, W. J., and Ruddle, F. H. (1998). Molecular evolution of Hox gene regulation: Cloning and transgenic analysis of the lamprey HoxQ8 gene. J. Exp. Zool. 280(1): 73-85.

Chesselet, M. F., Weiss, L., Wuenschell, C., Tobin, A. J., and Affolter, H. U. (1987). Comparative distribution of mRNAs for glutamic acid decarboxylase, tyrosine hydroxylase, and tachykinins in the basal ganglia: An in situ hybridization study in the rodent brain. J. Comp. Neurol. 262(1): 125-140.

Farmerie, T. A., Abbud, R. A., Budworth, P. R., Clay, C. M., Keri, R. A., McDowell, K. J., Wolfe, M. W., and Nilson, J. H. (1997). Characterization of the equine glycoprotein hormone alpha-subunit gene reveals divergence in the mechanism of pituitary and placental expression. Biol. Reprod. 57(5): 1104-1114.

Fiskerstrand, C., and Quinn, J. P. (1996). The molecular biology of preprotachykinin-A gene expression. Neuropeptides 30(6): 602-610.

Fiskerstrand, C. E., Lovejoy, E., Gerrard, L., and Quinn, J. P. (1999a). An intronic domain within the rat preprotachykinin-A gene con- taining a CCCT repetitive motif acts as an enhancer in differentiating embryonic stem cells. Neurosci. Lett. 263(2-3): 141-144.

Fiskerstrand, C. E., Newey, P., Ebrahimi, B., Gerrard, L., Harrison, P., McGregor, G. P., and Quinn, J. P. (1999b). Novel cell lines for the analysis of preprotachykinin A gene expression identify a repressor domain $3^{\prime}$ of the major transcriptional start site. Biochem. J. 341(Pt. 3): 847-852.

Gresch, P. J., and Walker, P. D. (1999). Serotonin-2 receptor stimulation normalizes striatal preprotachykinin messenger RNA in an animal model of Parkinson's disease. Neuroscience 93(3): 831-841.

Harlan, R. E., Garcia, M. M., and Krause, J. E. (1989). Cellular localization of substance $\mathrm{P}$ - and neurokinin A-encoding preprotachykinin mRNA in the female rat brain. J. Comp. Neurol. 287(2): 179-212.

Harmar, A. J., Mulderry, P. K., al-Shawi, R., Lyons, V., Sheward, W. J., Bishop, J. O., and Chapman, K. (1993). $3.3 \mathrm{~kb}$ of $5^{\prime}$ flanking DNA from the rat preprotachykinin gene directs high level expression of a reporter gene in microinjected dorsal root ganglion neurons but not in transgenic mice. Regul. Pept. 46(1-2): 67-69.

Hasenohrl, R. U., Jentjens, O., De Souza Silva, M. A., Tomaz, C., and Huston, J. P. (1998). Anxiolytic-like action of neurokinin substance $\mathrm{P}$ administered systemically or into the nucleus basalis magnocellularis region. Eur. J. Pharmacol. 354(2-3): 123-133.

Hogan, B., Beddington, R., Constantini, F., and Lacy, E. (1995). Manipulating the Mouse Embryo, Cold Spring Harbor Laborotory Press, Cold Spring Harbor, New York.

Hurd, Y. L., Keller, E., Sotonyi, P., and Sedvall, G. (1999). Preprotachykinin-A mRNA expression in the human and monkey brain: An in situ hybridization study. J. Comp. Neurol. 411(1): 56-72.

Iwasaki, Y., Kinoshita, M., Ikeda, K., Takamiya, K., and Shiojima, T. (1989). Trophic effect of various neuropeptides on the cultured ventral spinal cord of rat embryo. Neurosci. Lett. 101(3): 316-320.

Krasnova, I. N., Bychkov, E. R., Lioudyno, V. I., Zubareva, O. E., and Dambinova, S. A. (2000). Intracerebroventricular administration of substance P increases dopamine content in the brain of 6-hydroxydopamine-lesioned rats. Neuroscience 95(1): 113-117.

Krause, J. E., Chirgwin, J. M., Carter, M. S., Xu, Z. S., and Hershey, A. D. (1987). Three rat preprotachykinin mRNAs encode the neuropeptides substance P and neurokinin A. Proc. Natl. Acad. Sci. USA 84(3): 881-885.

Lamb, B. T., and Gearhart, J. D. (1995). YAC transgenics and the study of genetics and human disease. Curr. Opin. Genet. Dev. 5(3): 342348.

Liu, H., Mazarati, A. M., Katsumori, H., Sankar, R., and Wasterlain, C. G. (1999). Substance P is expressed in hippocampal principal neurons during status epilepticus and plays a critical role in the maintenance of status epilepticus. Proc. Natl. Acad. Sci. USA 96(9): 5286-5291.

Liu, M., Pleasure, S. L., Collins, A. E., Noebels, J. L., Naya, F. J., Tsai, M. J., and Lowenstein, D. H. (2000). Loss of BETA2/NeuroD leads to malformation of the dentate gyrus and epilepsy. Proc. Natl. Acad. Sci. 97(2): 865-870.

MacKenzie, A., and Quinn, J. (1999). A serotonin transporter gene intron 2 polymorphic region, correlated with affective disorders, has allele-dependent differential enhancer-like properties in the mouse embryo. Proc. Natl. Acad. Sci. USA 96(26): 15251-15255.

Magdaleno, S. M., and Curran, T. (1999). Gene dosage in mice-BAC to the future. Nat. Genet. 22(4): 319-320.

Maggio, J. E. (1988). Tachykinins. Annu. Rev. Neurosci. 11: 13-28.

Markie, D. (1996). Markers, selection and media in YAC cloning. In YAC Protocols (Markie, D., Ed.), Vol. 54. Humana Press, Totowa, NJ. McKee-Johnson, J. a. R., R. H. (1996). Fragmentation and integrative 
modification of YACs. In YAC Protocols (Markie, D., Ed.), Vol. 54. Humana Press, Totowa, NJ.

Mendelson, S. C., Fiskerstrand, C. E., and Quinn, J. P. (1998). A role for the octamer-binding protein in preprotachykinin-A gene expression. Neuropeptides 32(1): 79-85.

Montoliu, L., Umland, T., and Schutz, G. (1996). A locus control region at $-12 \mathrm{~kb}$ of the tyrosinase gene. Embo. J. 15(22): 6026-6034.

Morrison, C. F., McAllister, J., Dobson, S. P., Mulderry, P. K., and Quinn, J. P. (1994a). An activator element within the preprotachykinin-A promoter. Mol. Cell. Neurosci. 5(2): 165-175.

Morrison, C. F., McAllister, J., Lyons, V., Chapman, K., and Quinn, J. P. (1994b). The rat preprotachykinin-A promoter is regulated in PC12 cells by the synergistic action of multiple stimuli. Neurosci. Lett. 181(1-2): 117-120.

Nian, M., Drucker, D. J., and Irwin, D. (1999). Divergent regulation of human and rat proglucagon gene promoters in vivo. Am. J. Physiol. 277(4, Pt. 1): G829-G837.

Niederfuhr, A., Hummerich, H., Gawin, B., Boyle, S., Little, P. F., and Gessler, M. (1998). A sequence-ready 3-Mb PAC contig covering 16 breakpoints of the Wilms tumor/anirida region of human chromosome 11p13. Genomics 53(2): 155-163.

Noguchi, K., Morita, Y., Kiyama, H., Ono, K., and Tohyama, M. (1988). A noxious stimulus induces the preprotachykinin-A gene expression in the rat dorsal root ganglion: A quantitative study using in situ hybridization histochemistry. Brain Res. 464(1): 31-35.

Pernow, B. (1983). Substance P. Pharmacol. Rev. 35(2): 85-141.

Porcu, S., Kitamura, M., Witkowska, E., Zhang, Z., Mutero, A., Lin, C., Chang, J., and Gaensler, K. M. (1997). The human beta globin locus introduced by YAC transfer exhibits a specific and reproducible pattern of developmental regulation in transgenic mice. Blood 90(11): 4602-4609.

Quinn, J. P., McAllister, J., and Mendelson, S. (1995a). Multiple protein complexes, including AP2 and Sp1, interact with a specific site within the rat preprotachykinin-A promoter. Biochim. Biophys. Acta 1263(1): 25-34.

Quinn, J. P., Mendelson, S. C., Paterson, J. M., McAllister, J., and Morrison, C. F. (1995b). Transcriptional control of neuropeptide gene expression in sensory neurons, using the preprotachykinin-A gene as a model. Can. J. Physiol. Pharmacol. 73(7): 957-962.

Ragoussis, J. (1996). Restriction analysis of YACs. In YAC Protocols (Markie, D., Ed.), Vol. 54. Humana Press, Totowa, NJ.

Richfield, E. K., Maguire-Zeiss, K. A., Vonkeman, H. E., and Voorn, P. (1995). Preferential loss of preproenkephalin versus preprotachykinin neurons from the striatum of Huntington's disease patients [see comments]. Ann. Neurol. 38(6): 852-861.

Schedl, A., Grimes, B., and Monoliu L. (1996). YAC transfer by Microinjection. In YAC Protocols (Markie, D., Ed.), Vol. 54. Humana Press, Totowa, NJ.

Warden, M. K., and Young, W. S. d. (1988). Distribution of cells containing mRNAs encoding substance $\mathrm{P}$ and neurokinin $\mathrm{B}$ in the rat central nervous system. J. Comp. Neurol. 272(1): 90-113.

Zimmer, A., Zimmer, A. M., Baffi, J., Usdin, T., Reynolds, K., Konig, M., Palkovits, M., and Mezey, E. (1998). Hypoalgesia in mice with a targeted deletion of the tachykinin 1 gene. Proc. Natl. Acad. Sci. USA 95(5): 2630-2635.

Zweigerdt, R., Braun, T., and Arnold, H. H. (1997). Faithful expression of the Myf-5 gene during mouse myogenesis requires distant control regions: A transgene approach using yeast artificial chromosomes. Dev. Biol. 192(1): 172-180. 